\title{
Matching Conditions in the Einstein-Cartan Theory of Gravitation *
}

\author{
W. Arkuszewski \\ Institute of Astronomy, Polish Academy of Sciences. Warsaw, Poland

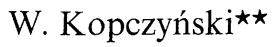 \\ Institute of Theoretical Physics, Warsaw University. Warsaw, Poland \\ V. N. Ponomariev \\ Department of Theoretical Physics, Moscow University, Moscow, USSR
}

\begin{abstract}
In this paper matching and junction conditions in the EinsteinCartan theory are presented. It is shown that a Weyssenhoff fluid sphere collapses to a singularity.
\end{abstract}

\section{Introduction}

In the general relativity theory two types of singularities are of a relevant physical interest. These are the singularities of cosmology and of gravitational collapse. In the framework of the Einstein-Cartan theory [1-4], being a modification of the classical theory of gravitation, cosmological models without singularities were constructed [5-9]; the problem of singularities of the second type is not clear. To solve this problem, as the first step, we investigate conditions which gravitational fields on a star's surface should satisfy.

Initial sections of this paper have an informative character. Section I contains a brief presentation of the Einstein-Cartan theory based on Trautman's approach $[3,4]$. In Section II, the investigation of discontinuities by means of differential forms is considered.

In Section III the matching conditions and their general implications are considered. We require the Einstein-Cartan equations to be satisfied in the distribution sense. The matching conditions are formulated as follows: (a) the metric tensor is continuous, (b) the spin tensor and (c) the energy-momentum tensor have at most "regular" discontinuities. Due to the previous requirement, condition (c) can be formulated in a form which is more convenient for applications: the second fundamental form with respect to the dynamical, assymetric, connection ${ }^{1}$ and the trace of the projected defect tensor ${ }^{2}$ are continuous. If spin vanishes,

* Supported in part by the National Science Foundation Grant GF-36217.

$\star \star$ Part of this work was done during the stay of the author in Department of Mechanics, Paris University VI and in Collège de France.

1,2 Definitions are given below. 
or if it is at least continuous, one can prove that the metric's first derivatives must be continuous in an admissible coordinate system, as it is usually stated in the general relativity theory [10]. The matching conditions imply the junction conditions, being restrictions on the behaviour of matter tensor (energy-momentum and spin tensors) on a match-hypersurface. In Section III, we formulate special junction conditions which the matter tensor of an internal gravitational field should satisfy to assure its prolongation into empty space. Whereafter, we show that these conditions are sufficient for an external, locally unique, vacuum gravitational field to exist. It appears that Prasanna's spherically symmetric static solutions [11] are inconsistent with the equations of the theory because they contradict these junction conditions.

In the last section, we apply the junction conditions in a special case of spherical symmetry. We consider, as the internal gravitational field, the Friedmann-like solution for the Weyssenhoff fluid found by one of the authors [12]. The junction conditions and the energy domination condition $(p \leqq \varepsilon)$ imply that the gravitational collapse of the corresponding star leads to a singularity. However, the case of spherical symmetry is not physically interesting (at least, because of the fact that the pseudovector of spin density has a radial distribution which gives a space-like singularity [4]). To solve the singularity problem of the collapse type for Weyssenhoff fluid stars it seems necessary to construct a more realistic, non-spherical, model of such a star.

\section{The Einstein-Cartan Equations}

In the Einstein-Cartan theory, gravitation is described by the metric tensor $g_{i j}$ (we use the signature +---) and the metric connection $\omega^{i}{ }_{j}=\Gamma^{i}{ }_{j k} d x^{k}$. The defect ${ }^{3}$ $x_{j}^{i}=x_{j k}^{i} d x^{k}$ of the connection $\omega^{i}{ }_{j}$ is the difference

$$
x_{j}^{i}=\omega^{i}{ }_{j}-\tilde{\omega}^{i}{ }_{j}
$$

where $\tilde{\omega}_{j}^{i}$ is the Riemannian connection associated with $g_{i j}$. Due to the metricity of $\omega^{i}{ }_{j}$, the defect is skewsymmetric,

$$
x_{i j}+x_{j i}=0 \text {. }
$$

The torsion $Q^{i}{ }_{j k}=\Gamma^{i}{ }_{k j}-\Gamma^{i}{ }_{j k}$ of the connection $\omega^{i}{ }_{j}$ is related to the defect by the relation

$$
Q^{i}{ }_{j k}=x^{i}{ }_{k j}-x^{i}{ }_{j k} .
$$

The curvature $\Omega^{i}{ }_{j}=\frac{1}{2} R^{i}{ }_{j k l} d x^{k} \wedge d x^{l}$ of the connection $\omega^{i}{ }_{j}$ is defined by

$$
\Omega_{j}^{i}=d \omega^{i}{ }_{j}+\omega^{i}{ }_{k} \wedge \omega^{k}{ }_{j} \text {. }
$$

It is convenient to use the following collection of pseudotensor differential forms:

$$
\begin{aligned}
& \eta_{i j k}=d x^{l} \eta_{i j k l}, \quad \eta_{i j}=\frac{1}{2} d x^{k} \wedge \eta_{i j k}, \\
& \eta_{i}=\frac{1}{3} d x^{j} \wedge \eta_{i j}, \quad \eta=\frac{1}{4} d x^{i} \wedge \eta_{i},
\end{aligned}
$$

3 Hehl $[1,2]$ uses the term "contortion". 
where $\eta_{i j k l}$ is the Levi-Civita completely skewsymmetric pseudotensor, $\eta_{0123}=$ $(-\operatorname{det} g)^{1 / 2}$.

The energy-momentum and the spin of matter are sources of the gravitational field in the theory. They are represented by the 3 -forms $t_{i}=t_{i}^{j} \eta_{j}$ and $s_{i j}=s^{k}{ }_{i j} \eta_{k}$ respectively.

The relations

$$
\begin{aligned}
& x^{i}{ }_{j k}=-4 \pi G\left(s^{i}{ }_{j k}+s_{k j}{ }^{i}+s_{j k}{ }^{i}-\delta_{k}{ }^{i}{ }^{l}{ }_{j l}+g_{k j} s^{l i}{ }^{i}\right), \\
& G^{i}{ }_{j}=8 \pi G t^{i}{ }_{j},
\end{aligned}
$$

where $G_{j}^{i}=R_{k j}^{k i}-\frac{1}{2} \delta_{j}^{i} R_{k l}^{k l}$, are called the Einstein-Cartan equations. Equation (3) are equivalent to

$$
\frac{1}{2} \eta_{i j}{ }^{k} \wedge \Omega^{j}{ }_{k}=-8 \pi G t_{i} .
$$

If the spin of matter is zero, the defect of $\omega_{j}^{i}$ vanishes and Eqs. (3) become the Einstein equations. In particular, the vacuum field Eqs. (3) are the same as in the classical theory of gravitation.

\section{Discontinuities and Differential Forms}

Let the space-time $\mathscr{X}$ be a differential manifold of the class $C^{2}$, piecewise of the class $C^{4}[10]$. It means that there exists a family of isolated hypersurfaces in $\mathscr{X}$ such that any transition function $\eta \circ \xi^{-1}$ between admissible, local coordinate systems $\xi=\left(\xi^{i}\right)$ and $\eta=\left(\eta^{i}\right)$ is of the class $C^{2}$ everywhere, is of the class $C^{4}$ outside of the hypersurfaces and its third and fourth derivatives tend uniformly to finite left and right limits on the hypersurfaces. It is enough to consider the single hipersurface $\Sigma$, given locally by the equation $x=0$.

It is convenient to describe the differential properties of geometrical objects on $\mathscr{X}$ using the local admissible coordinate system $\left(x, x^{\alpha}\right)$, where $\left(x^{\alpha}\right), \alpha=1,2,3$, defines a local coordinate system on any hypersurface of constant coordinate $x$, in particular on $\Sigma$.

We say that the function $f: \mathscr{X} \rightarrow \mathbb{R}$ is of the class $C_{q}^{-1}(q \leqq 4)$, if the function $\left.f^{\prime}\right|_{x-\Sigma}$ is of the class $C^{q}$ and its derivatives, from the zero to the $q$-th inclusive, tend uniformly to finite left and right limits on $\Sigma$. If, moreover, $f$ is of the class $C^{p}(p \leqq 2)$ everywhere, we say that this function is of the class $C^{p}$. The function $f$ of the class $C_{q}^{p}$ defines on $\Sigma$ the functions $f_{+}, f_{-}$and $[f]$ of the class $C^{4}$ :

$$
\begin{aligned}
& f_{+}\left(x^{\alpha}\right)=\lim _{x \rightarrow+0} f\left(x, x^{\alpha}\right), \\
& f_{-}\left(x^{\alpha}\right)=\lim _{x \rightarrow-0} f\left(x, x^{\alpha}\right), \\
& {[f]=f_{+}-f_{-} .}
\end{aligned}
$$

Let us consider derivatives in the distribution sense of the function $f$ which is of the class $C_{q}^{-1}$, where $q \geqq 1$. The derivatives $\partial f / \partial x^{\alpha}$ are regular distributions (functions) of the class $C_{q-1}^{-1}$ and the following relation holds:

$$
\left[\partial f / \partial x^{\alpha}\right]=\partial[f] / \partial x^{\alpha} .
$$


The derivative $\partial f^{\prime} / \partial x$ is, in general, a singular distribution of the $\delta$-type, whereas

$$
\partial f / \partial x\left(x, x^{\alpha}\right)-[f]\left(x^{\alpha}\right) \delta(x)
$$

is a regular distribution of the class $C_{q-1}^{-1}$.

We say that a geometrical object (like a tensor field and an affine connection) on $\mathscr{X}$ is of the class $C_{q}^{p}$ (where $1 \geqq p \geqq-1,3 \geqq q \geqq 0$ ), if its coefficients in any admissible coordinate system are of the class $C_{q}^{p}$. In particular, this definition can be applied to differential forms. If $\varphi$ is a differential form of the class $C_{q}^{-1}$, the properties (3) and (4) may be transferred as follows:

$$
d x \wedge[d \varphi]=d x \wedge d[\varphi]
$$

and

$$
d \varphi\left(x, x^{\alpha}\right)=d x \wedge[\varphi]\left(x^{\alpha}\right) \delta(x)+\psi\left(x, x^{\alpha}\right),
$$

where $\psi$ is a regular distribution of the class $C_{q-1}^{-1}$.

We see that the condition $d x \wedge[\varphi]=0$ is necessary and sufficient to assure regularity of $d \varphi$.

\section{The Matching Conditions}

We assume that:

(a) The metric tensor $g_{i j}$ is of the class $C_{\mathbf{3}}^{0}$

From this assumption, it follows at once that the Riemannian connection $\tilde{\omega}_{j}^{i}$ is of the class $C^{-1}$.

Further on, we shall assume that the hypersurface $\Sigma$ is timelike. The function $x$ defines the local vector field $n_{i}=\partial_{i} x /|\nabla x|$, which is orthogonal to $\Sigma$ and normalized, $n_{i} n^{i}=-1$. This vector field is of the class $C_{3}^{0}$. Let us define the operator $h_{j}^{i}$ (of the class $C_{3}^{0}$ ) of the orthogonal projection on the family of hypersurfaces $x=$ const,

$$
h^{i}{ }_{j}=\delta^{i}{ }_{j}+n^{i} n_{j} .
$$

If $T$ is a geometric object defined in a neighbourhood of $\Sigma, \bar{T}$ denotes its projection on the family $x=$ const.

In particular,

$$
\overline{\tilde{\omega}}_{j}^{i}=h_{l}^{i} h_{j}^{m} h_{k}^{n} \tilde{\Gamma}_{m n}^{l} d x^{k}
$$

is the Riemannian connection on each of these hypersurfaces associated with $\bar{g}_{i j}=h_{i j}$. The Riemannian connection $\overline{\tilde{\omega}}_{j}^{i}$ is algebraically dependent on $\partial \bar{g}_{i j} / \partial x^{\alpha}$ and $\bar{g}_{i j}$, so it is of the class $C_{2}^{0}$,

$$
\left[\overline{\tilde{\omega}}^{i}{ }_{j}\right]=0 \text {. }
$$

We also assume that:

(b) the spin tensor $s_{i j}^{k}$ is of the class $C^{-1}$.

The conditions (a) and (b) imply that the defect $x^{i}{ }_{j}$, the torsion $Q^{i}{ }_{j k}$ and the connection $\omega^{i}{ }_{j}$ are of the class $C^{-\frac{1}{2}}$. From Eqs. (1) and (6), we get

$$
\left[\bar{\omega}^{i}{ }_{j}\right]=\left[\bar{x}^{i}{ }_{j}\right] \text {. }
$$


The formula

$$
N_{i}=\overline{\nabla_{j} n_{i}} d x^{j} \equiv \overline{d n_{i}}-\overline{\omega^{k}{ }_{i} n_{k}}
$$

defines the second fundamental form $N_{i}=N_{i j} d x^{J}$ of any hypersurface $x=\mathrm{const}$ with respect to the connection $\omega_{\tilde{N}^{i}} . N_{i}$ is related to the Riemannian, symmetric, second fundamental form $\tilde{N}_{i}=\tilde{N}_{i j} d x^{j}$ by the equation

$$
N_{i}=\tilde{N}_{i}-\overline{\varkappa_{i}^{k} n_{k}} \text {. }
$$

Let us assume that:

(c) the energy-momentum tensor $t^{j}{ }_{i}$ is of the class $C^{-1}$

If the Einstein-Cartan field equation ( $\left.3^{\prime}\right)$ are satisfied in the distribution sense, (c) is equivalent to

$$
\frac{1}{2} \eta_{i j}{ }^{k} \wedge d x \wedge\left[\omega^{j}{ }_{k}\right]=0 \text {. }
$$

Let us decompose the form $d x \wedge \omega^{i}{ }_{j}$ as

$$
d x \wedge \omega^{i}{ }_{j}=d x \wedge\left(\bar{\omega}^{i}{ }_{j}-\omega^{k}{ }_{j} n_{k} n^{i}-\overline{\omega^{i}{ }_{k} n^{k}} n_{j}+\omega^{k}{ }_{l} n_{k} n^{l} n^{i} n_{j}\right)
$$

and observe that the form

$$
d x \wedge\left(\omega_{i j}+\omega_{j i}\right)=d x \wedge d g_{i j}
$$

is continuous. As a consequence of this, condition (10) reduces to the following two conditions:

$$
\begin{aligned}
& \frac{1}{2} \eta_{i j}{ }^{k} \wedge d x \wedge\left[\bar{\omega}^{j}{ }_{k}\right]=0, \\
& \frac{1}{2} \eta_{i j}{ }^{k} \wedge d x \wedge\left[\overline{\left.\omega^{l}{ }_{k} n_{l} n^{j}\right]=0 .}\right.
\end{aligned}
$$

Taking into account Eqs. (7) and (8), we can write the above conditions as

$$
\begin{aligned}
& \frac{1}{2} \eta_{i j}{ }^{k} \wedge d x \wedge\left[\bar{x}^{j}{ }_{k}\right]=0 \\
& \frac{1}{2} \eta_{i j}{ }^{k} \wedge d x \wedge\left[N_{k}\right] n^{j}=0
\end{aligned}
$$

or equivalently

$$
\begin{aligned}
& {\left[\bar{x}^{k}{ }_{i k}\right]=0,} \\
& {\left[N_{i j}\right]=0 .}
\end{aligned}
$$

If spin vanishes, condition (11) becomes trivial, whereas condition (12) assures regularity of the curvature $\Omega^{i}$. However, in general, regularity of the energy momentum tensor $t^{j}{ }_{i}$ is insufficient to assure regularity of $\Omega^{i}$, because the form $d x \wedge \omega^{i}$

is continuous if and only if Eq. (12) and the equation

$$
\left[\bar{\varkappa}^{k}{ }_{i j}\right]=0 \text {, }
$$

instead of Eq. (11), are satisfied.

In the special coordinate system $\left(x, x^{\alpha}\right)$, the continuity condition of the symmetric part $N_{(i j)}$ of the second fundamental form reduces to

$$
\left[\partial g_{\alpha \beta} / \partial x\right]=\left(2 / g^{00}\right)\left[x_{(\alpha \beta)}^{0}\right] \text {. }
$$


Because the derivatives $\partial g_{i j} / \partial x^{\gamma}$ are continuous and the derivatives $\partial g_{0 i} / \partial x$ can be made continuous by a convenient (and admissible) choice of a coordinate system, one can say that Eq. (13) determines discontinuities of first derivatives of the metric tensor.

The continuity condition of the skewsymmetric part $N_{[i j]}$ of the second fundamental form can be written, due to Eq. (9), as

$$
\left.\overline{n_{k} x^{k}[i j]}\right]=0 \text {. }
$$

Using formula (2) one can check that conditions (11) and (14) are equivalent to the equation

$$
\left[n_{k} s_{i j}^{k}\right]=0 \text {. }
$$

We have reduced assumption (c) to the relations (13) and (15). Equation (15) gives a restriction on possible spin discontinuities, while Eq. (13) expresses discontinuities of the metric tensor derivatives by spin discontinuities. When the spin tensor is continuous, we obtain Lichnerowicz's matching conditions [10]. If Eq. (13) is satisfied, the symmetric part $t_{(i j)}$ of the energy-momentum tensor is a regular distribution. Its skewsymmetric part $t_{[i j]}$ is regular, if Eq. (15) is satisfied; this can be easily seen from the generalized angular momentum conservation law $[3,4]$.

On the other hand, from the generalized energy-momentum conservation law $[3,4]$ the following equality can be deduced:

$$
8 \pi G\left[n_{j} t_{i}^{j}\right]+\left[\bar{\chi}_{j k i}\right] n_{l} x^{l j k}+\frac{1}{2} n_{i}\left[\bar{\chi}_{j k l} \bar{\varkappa}^{l k j}\right]=0 .
$$

We say that a gravitational field given in the region $x<0$ matchs a gravitational field given in the region $x>0$, if conditions (a), (b) and (c) are satisfied in any admissible coordinate system. These conditions imply that the matter tensors $t^{j}{ }_{i}$ and $s_{i j}^{k}$, being sources of those fields, satisfy the junction conditions (15) and (16). One can prove that the junction conditions are the only independent restrictions imposed on the jumps $\left[t^{j}{ }_{i}\right]$ and $\left[s^{k}{ }_{i j}\right]$ of the matter tensors by conditions (a), (b), (c).

\section{The Junction Conditions as Boundary Conditions for the Vacuum Einstein Equations}

Let the metric tensor $g_{i j}$, corresponding to the matter tensors $t^{j}{ }_{i}$ and $s^{k}{ }_{i j}$, be given in the region $x<0$, limited by the hypersurface $\Sigma$. To assure existence, on the other side of $\Sigma$, of a vacuum gravitational field matching this internal metric, the matter tensors must satisfy the special junction conditions:

$$
n_{k} s_{i j}^{k} l_{-}=0
$$

and

$$
\left.8 \pi G n_{j} t^{j}\right|_{-}+\left.\frac{1}{2} n_{i} \bar{x}_{j k l} \bar{\varkappa}^{l k j}\right|_{-}=0 .
$$

These formulae follow from the junction conditions (15) and (16). 
Let us suppose that Eqs. (17) and (18) are fulfilled. Then one can solve the Einstein vacuum equations,

$$
G_{i}^{j}=0, \text { for } x>0,
$$

with the boundary conditions

$$
\begin{aligned}
& \left.g_{i j}\right|_{+}=\left.g_{i j}\right|_{-}, \\
& \partial g_{\alpha \beta} /\left.\partial x\right|_{+}=\partial g_{\alpha \beta} /\left.\partial x\right|_{-}-2 x_{(\alpha \beta)}^{0} /\left.g^{00}\right|_{-},
\end{aligned}
$$

because the constraint conditions, $\left.G^{0}\right|_{+}=0$, are satisfied. Due to Eqs. (17), (18), (19) and (20) the above constructed external metric matchs the internal metric. Moreover, this external metric is a (geometrically) unique vacuum metric, determined in a neighbourhood of $\Sigma$, matching the given internal $g_{i j}[10]$.

\section{Collapse of a Weyssenhoff Fluid Sphere}

The energy-momentum and spin tensors of the Weyssenhoff fluid $[13,14]$ are defined as follows:

$$
\begin{aligned}
& t^{i}{ }_{j}=u^{i}\left(P_{j}+p u_{j}\right)-p \delta_{j}^{i}, \\
& s_{j k}^{i}=u^{i} \eta_{j k l m} u^{l} S^{m}, \quad u^{m} S_{m}=0 .
\end{aligned}
$$

The vector $u_{i}$ is the fluid velocity. The vector $P_{j}$ and the pseudovector $S^{m}$ represent the energy-momentum density and the spin density respectively. The scalar $p$ is the pressure of the fluid.

In the case of the Weyssenhoff fluid the special junction conditions (17) and (19) take on the form

$$
\begin{aligned}
& \left.n_{i} u^{i}\right|_{-}=0, \\
& \left.p\right|_{-}=\left.2 \pi G\left(n_{i} S^{i}\right)^{2}\right|_{-} .
\end{aligned}
$$

Condition (21) is the same as in classical relativistic hydrodynamics, while condition (22) is different from that case. The pressure $p$ on the surface of a Weyssenhoff fluid star does not necessarilly vanish; it can be zero only if the pseudovector $S^{i}$ is orthogonal to this surface. One can check that in this case the curvature of the connection $\omega_{j}^{i}$ is continuous across the star's surface.

Let us apply conditions (21) and (22) to the simple case of spherical symmetry. As the internal gravitational field, the solution of the Einstein-Cartan equations reported in $[4,12,15]$ and generalized to the case of nonvanishing pressure, is taken. The metric tensor of this solution is of the Robertson-Walker type,

$$
d s^{2}=d t^{2}-R^{2}(t)\left(\left(1-k r^{2}\right)^{-1} d r^{2}+r^{2}\left(d \delta^{2}+\sin ^{2} \delta d \varphi^{2}\right)\right),
$$

where $k=0, \pm 1$. The pseudovector $S^{i}$ has only a radial component. The spin density $S=\left(-S_{i} S^{i}\right)^{1 / 2}$, the energy density $\varepsilon=u^{i} P_{i}$ and the pressure $p$ depend only on the time $t$. The Einstein-Cartan equations give:

$$
\begin{aligned}
& 8 \pi G\left(\varepsilon-2 \pi G S^{2}\right)=3\left(\dot{R}^{2}+k\right) R^{-2}, \\
& 8 \pi G\left(p-2 \pi G S^{2}\right)=-2 \ddot{R} R^{-1}-\left(\dot{R}^{2}+k\right) R^{-2}, \\
& S R^{3}=\mathrm{const},
\end{aligned}
$$

where a dot denotes differentiation with respect to $t$. 
The quantities $[15,16]$

$$
\begin{aligned}
& \varepsilon_{\text {eff }}=\varepsilon-2 \pi G S^{2}, \\
& p_{\text {eff }}=p-2 \pi G S^{2}
\end{aligned}
$$

play the same role in Eqs. (23) as $\varepsilon$ and $p$ play in the equations of the Einsteinian cosmology. The junction conditions (21) and (22) imply that

$$
p_{\text {eff }}=\left.p_{\text {eff }}\right|_{-}=\left.p\right|_{-}-\left.2 \pi G\left(n_{i} S^{i}\right)^{2}\right|_{-}=0,
$$

so we have to solve the modified Friedmann equations (23) with vanishing effective pressure. The first integral of these equations is well known,

$$
\frac{1}{2} \dot{R}^{2}-M G / R=-k / 2,
$$

where the constant $M$ is defined by

$$
M=(4 / 3) \pi R^{3} \varepsilon_{\text {eff }} .
$$

If $M>0$, or if $M=0$ and $\dot{R}\left(t_{0}\right) \neq 0$, the solution $R(t)$ of Eq. (24) must reach zero. We conclude that the gravitational field of this collapsing Weyssenhoff fluid star must be singular if $\varepsilon_{\text {eff }} \geqq 0$ or, equivalently, if $\varepsilon \geqq p$.

Acknowledgement. One of the authors (W.K.) thanks Prof. Y.Choquet-Bruhat for a discussion and careful reading of a manuscript.

\section{References}

1. Hehl,F.W.: Spin und Torsion in der allgemeinen Relativitätstheorie oder die Riemann-Cartansche Geometrie der Welt. Habil. thesis, Techn. Univ. Clausthal (1970)

2. Hehl, F.W.: GRG J. 4, 333-349 (1973)

3. Trautman, A.: Bull. Polon. Acad. Sci., sér. sci. math. astr. et phys. 20, 185-190, 503-506 (1972)

4. Trautman,A.: On the structure of the Einstein-Cartan equations (with an Appendix by W. Kopczyński). Preprint, Warsaw University IFT/72/13

5. Kopczyński, W.: Phys. Lett. 43A, 63-64 (1973)

6. Trautman, A.: Nature (Phys. Sci.) 242, 7-8 (1973)

7. Tafel, J.: Phys. Lett. 45A, 341-342 (1973)

8. Tafel, J.: A class of cosmological models with torsion and spin. Will be published in Acta Phys. Polon

9. Kerlick, D.: A mixmaster universe with torsion. To be published

10. Lichnerowicz, A.: Théories relativistes de la gravitation et de l'électromagnétisme. Paris: Masson et Cie 1955

11. Prasanna,A.R.: Static Fluid Spheres in Einstein-Cartan Theory. Preprint, ICTP Trieste $\mathrm{IC} / 73 / 120$

12. Kopczyński, W.: Phys. Lett. 39A, 219-220 (1972)

13. Weyssenhoff, J., Raabe, A.: Acta Phys. Polon. 9, 7-31 (1947)

14. Halbwachs, F.: Théories relativistes des fluides à spin. Paris: Gauthier-Villars 1960

15. Hehl, F. W., v. d. Heyde, P.: Ann. Inst. Henri Poincaré A19, 179-196 (1973)

16. Arkuszewski, W., Kopczyński, W., Ponomariev, V. N.: Ann. Inst. Henri Poincaré (in print)

Communicated by J. Ehlers 